\title{
A Comparative Study of Memory, Attention, Cognition and Oestradiol in Pre and Postmenopausal Women in Bangladesh
}

\author{
Kaniz Rabeya ${ }^{1}$, Mamtaz Mahal Neela ${ }^{2}$, Ragib Ahsan ${ }^{1,3}$, Bishyajit Kumar Biswas ${ }^{1}$ \\ and Md. Monir Hossain ${ }^{1,4}$
}

\author{
${ }^{1}$ Department of Pharmacy, Jagannath University, Dhaka-1100, Bangladesh \\ ${ }^{2}$ Institute of Social Welfare and Research, University of Dhaka, Dhaka-1205 \\ ${ }^{3}$ Department of Biological Science, College of Natural Science, University of Ulsan, Ulsan, 44610, South Korea \\ ${ }^{4}$ Department of Biomedical Sciences, City University of Hong Kong, Kowloon Tong, Hong Kong SAR
}

(Received: August 15, 2020; Accepted: December 26, 2020; Published (web): January 28, 2021)

\begin{abstract}
Memory, attention, and cognitive dysfunction are psychopathological conditions which most commonly occur after menopause. Different clinical studies revealed a shred of substantial evidence that oxidative stress and estrogen are interlinked in various cognitive dysfunction, including memory impairment, agerelated dementia, and Alzheimer's disease. There is a higher chance of developing cardiovascular disease after menopause. Sharp declines in concentrations of circulating estradiol and estrone are associated with menopause. Estrogen replacement therapy (ERT) enhances the blood circulation to the hippocampus and cortex, providing the optimum environment for the growth and survival of cholinergic neurons. Hence, it improves hippocampal neuron density and ultimately contributes to synaptic plasticity in the hippocampus enhances short and long-term memory. In this study, we assessed memory, attention, and cognition function between pre- and post-menopausal groups. After preliminary screening and applying exclusion criteria, fifteen premenopausal women and fifteen postmenopausal women were finally selected. Different neuropsychological tests such as logical memory test, digit span test, letter cancellation test, trail making test and Stroop test were performed to evaluate the memory, attention, and cognition status. Blood estradiol level was also assessed by using commercial kits. Significant difference $(\mathrm{p}<0.05)$ was found in LM-II in logical memory test, digit span test (backward), letter cancelation test, TMT-B in trail making test, the score of part $\mathrm{C}$ in stroop test between premenopausal and postmenopausal women. Serum oestradiol concentration $(\mathrm{pg} / \mathrm{ml})$ was significantly lower $(\mathrm{p}<0.001)$ in postmenopausal women $(44.18 \pm 10.52)$ than premenopausal women $(175.48 \pm 43.20)$. The current study demonstrates the memory decline and cognitive dysfunction in postmenopausal women and there is a significant difference in estradiol level between pre and postmenopausal women. Estrogen has many neurotrophic actions in the brain and helps to improve memory and cognition. Therefore, estrogen replacement therapy, dietary supplements or a drug having an agonistic effect on estrogen receptors might improve the status of memory, attention, and cognitive function in postmenopausal women.
\end{abstract}

Key words: Memory, attention, cognition, oestradiol, menopause.

\section{Introduction}

Bangladesh is a developing country in South East Asia. Most of the women have less consciousness about menopausal symptoms effect on the quality of life. "Menopause" is derived from two Greek words meno (month) and pause (to end). Women who had not menstruated within the previous 12 months were categorized as postmenopausal. The ages at which menopause occurs varies widely, ranging from the late thirties to late fifties. Most commonly, menopause occurred between ages 48 and 55 y (Henderson, 2000). Sharp declines in concentrations of circulating estradiol and estrone are associated with menopause, which has a potential impact on central nervous system function (Henderson, 2000). Hot flushes, osteoporosis,

Corresponding author: Md. Monir Hossain; E-mail: mmhossain4-c@my.cityu.edu.hk

DOI: https://doi.org/10.3329/bpj.v24i1.51632 
increased risk of cardiovascular diseases are the most common and noticeable symptoms of loss of ovarian hormones. Apart from the estrogenic action in the hypothalamus affecting ovulation and reproductive behavior, it is now apparent that the brain is one of the organs that suffer greatly from estrogen loss after surgical or natural menopause. Moreover, many women sufferings from difficulties in remembering names, a deficit in motor coordination, depression, and anxiety (Cersosimo and Benarroch, 2015).

Memory decline is a very common complaint throughout the menopausal transition (Woods et al., 2000). Alzheimer's disease (AD) is the most common neurodegenerative disease, comprising higher than $50 \%$ of all dementia types (Plassman et al., 2007). Women are twice more prone to AD due to increased longevity, the sex difference in brain size (Zandi et al., 2002). There is a higher possibility of developing $\mathrm{AD}$ in postmenopausal women than their male counterparts. Cognitive abilities deteriorate across aging in humans, mostly for women. Apart from aging, ovarian production of estrogen and progesterone markedly decreased after menopause. The previous report revealed that $62-70 \%$ of postmenopausal women report noticeable cognition deficits (Betti et al., 2001; Davey, 2013). Neurotrophic action of estrogen has been found in areas involved in memory and cognition (Tang et al., 1996). Studies showed that 17-beta estradiol protects against beta-amyloid-induced damage and tau-related changes (Small et al., 2006). Furthermore, magnetic resonance imaging (MRI) studies revealed that 17beta estradiol increases the blood flow to the hippocampus and cortex (Maki and Resnick, 2000; Persad et al., 2009). Allowed to promote the growth and survival of cholinergic neurons, hippocampal neurons become dense which finally contributes synaptic plasticity in the hippocampus and enhance the short and long-term memory (Luine, 1985).

Oxidative stress is the main culprit associated with the pathogenesis of numerous cognitive dysfunction, such as memory impairment, age-related dementia, Alzheimer's disease (Liu and Zhang, 2012; Xie et al., 2012; Clausen et al., 2012). Free radical oxidation of Glutathione (GSH) and the unsaturated fatty acids are commonly found in cognitive diseases (Ali, 2004). Free radical scavengers and antioxidants inhibit the progression of neurodegenerative disorders like dementia, Alzheimer's, etc. as suggested in these cases (Gibson and Huang, 2005; Stuchbury and Münch, 2005). Estrogen decreases the generation of oxygen free radicals by stabilizing membrane potential and prevent adenosine triphosphate (ATP) depletion (Wang et al., 2001). Estrogenic action on mood, locomotor activity, pain sensitivity, and cognition are mediated by binding nuclear intracellular receptor estrogen receptor alpha (ER-alpha) and estrogen receptor beta (ER-beta) on basal forebrain, hippocampus, cerebral cortex, midbrain raphe and spinal cord (Shughrue et al., 2000).

It is well revealed that every menopausal symptom impacts the Quality of Life (QOL) of menopausal women (Dhillon et al., 2006). Comprehensive research studies were conducted about the symptoms related to menopause in western countries. Whereas very little data is available from developing countries, especially South-East Asia. In this study, we assess memory, cognition, attention, and estradiol level in women who were in postmenopausal and in women who underwent regular menstruation.

\section{Materials and Methods}

Participants: Eighteen naturally postmenopausal women and sixteen premenopausal women with regular menstruation were enrolled randomly, and ultimately, fifteen volunteers in both groups took part in the experiment till last. Three postmenopausal participants found the test monotonous and, hence, did not participate in the research. One participant from premenopausal group was withdrawn from the study as she was colour-blind and not eligible to participate in the cognition test (Stroop test). Written informed consent was collected from each volunteer prior to the study. To evaluate the health conditions, participants were introduced to a complete set of medical health questions before selecting 
participants. All subjects were questioned and involved in the study if they met the following standards: (1) no history of preceding neuropathological story, (2) no history of hospital admission for psychiatric illness, (3) no previous engagement with drug or alcohol abuse. Those who had hypertension, hyperlipidemia, cardiovascular diseases, diabetes, history of cancer, hypothyroidism were excluded from the study. Women with apparent difficulties in speaking or understanding and surgically induced menopause were also excluded. Intake of any sex steroids during the last three months was not allowed. Subjects were advised to take standard dietary during the study period and should avoid caffeine or caffeine-related products before $12 \mathrm{~h}$ of the study. There is a nominal difference in Educational background and body-mass within the selected participants. The demographic information of all volunteers is presented in Table 1.

Table 1. Demographic data of the volunteers.

\begin{tabular}{|c|c|c|c|c|c|c|c|}
\hline Group & Age & Weight & $\begin{array}{l}\text { Height } \\
(\mathrm{cm})\end{array}$ & $\begin{array}{c}\text { Blood } \\
\text { pressure } \\
\text { (systolic) }\end{array}$ & $\begin{array}{c}\text { Blood } \\
\text { pressure } \\
\text { (diastolic) }\end{array}$ & $\begin{array}{c}\text { Education } \\
\text { year } \\
\text { (completed) }\end{array}$ & $\begin{array}{c}\text { Menopause } \\
\text { year }\end{array}$ \\
\hline Group A (Pre-menopause) & $22 \pm 3$ & $54 \pm 4.25$ & $156 \pm 2.76$ & $120 \pm 4.89$ & $80 \pm 7.18$ & $14 \pm 1.08$ & \\
\hline Group B (Post-menopause) & $52 \pm 2$ & $59.5 \pm 3.62$ & $154 \pm 2.31$ & $120 \pm 7.32$ & $80 \pm 9.88$ & $14 \pm 1.03$ & $5 \pm 1.03$ \\
\hline
\end{tabular}

\section{Test of memory}

Logical memory (LM): Immediate and delayed memory was measured by Logical memory test. A subtest of the Wechsler memory scale (Wechsler, 1997). A brief story was told to the volunteers, and later they were asked to recall the story twice to evaluate immediate memory (Logical Memory I, LM-I), and delayed memory after 30-min delay (Logical Memory II, LM-II).

Digit span test (DST): Digit span test (DST), a subtest of the Wechsler intelligence scale, was adopted to evaluate simple verbal working memory (Wechsler, 1987). There is two major part of this test, namely, digits forward (DSTF) and digits backward (DSTB). In the beginning, strings of digits were read aloud (e.g., 248 ) to the volunteers. The length of each string in the forward test is from three to nine and two to eight digits for the backward test. After that, the participant was asked to recall the string. Two successive incorrect strings reproduced by the participant in each subset, such as Forward and Backward was considered as the end of the test. Also, the trial ended when a participant successfully reproduced a full digit number. In the end, the sum of the maximal digit numbers that the participant can recall from forward and backward testing was measured, and the total score of each volunteer was determined.

\section{Attention test}

Letter cancellation test (LCT): Visual search, scanning, and attention of each volunteer were measured by the letter cancellation test (LCT) (Benton, 1968). In this test, a number of rows of letters were randomly placed on a sheet with a designated target letter. Performances were scored according to the number of correct responses (LCTC) test (LCTT). by the participant and the time taken to complete the

Trail making test (TMT): Trail-making test (TMT) Visual search, scanning, speed of processing, attention, mental flexibility, and executive functions were evaluated by Trail-making test (Reitan et al., 1985). TMT test is divided into two parts- TMTA and TMTB. Twenty-five encircled numbers distributed on a sheet of paper need to be drawn a line sequentially in TMTA part. Task requirements are similar for TMTB except for the fact that the person must alternate between numbers and letters (e.g., 1, A, 2, B, 3, C, etc.). The score on each part 
was evaluated by calculating the amount of time required to complete the task.

\section{Cognition test}

Stroop test (ST): The stroop color-Word testVictoria version (VST), originally developed by stroop (Regard, 1981; Stroop, 1935; Macleod, 1991; Graf et al., 1995; Verhaeghen et al., 1998) was used to measure selective attention and cognitive flexibility.

\section{Determination of oestradiol}

Commercially available Enzyme Linkedimmuno-sorbent Assay kit (ELISA), (Daniel et al., 2011) was used for the quantitative determination of 17-beta oestradiol.

Statistical analysis: For the data analysis, the statistical software package SPSS was used. An independent sample t-test was used to evaluate the significance level between premenopausal and postmenopausal groups. Here, $\mathrm{p}>0.05$ was considered as significant. Data were expressed as mean \pm standard deviation (SD).

Table 2. Neuropsychological test.

\begin{tabular}{|c|c|c|c|c|}
\hline \multicolumn{3}{|c|}{ Neuropsychological test } & \multicolumn{2}{|c|}{$\begin{array}{l}\text { ( } \mathrm{n}=15 \text { in each group and values are expressed } \\
\text { as mean } \pm \mathrm{SD})\end{array}$} \\
\hline & & & $\begin{array}{l}\text { Pre-menopause } \\
\text { (Group A) }\end{array}$ & $\begin{array}{l}\text { Post-menopause } \\
\text { (Group B) }\end{array}$ \\
\hline \multirow[t]{5}{*}{ Memory test } & \multirow[t]{2}{*}{ Logical memory (LM) } & LM-I & $10.07 \pm 3.00$ & $8.08 \pm 2.32$ \\
\hline & & LM-II & $10.08 \pm 2.84$ & $7.07 \pm 2.00 *$ \\
\hline & \multirow{3}{*}{ Digit span test (DST) } & Forward & $6.77 \pm 1.69$ & $5.86 \pm 1.10$ \\
\hline & & Backward & $5.55 \pm 1.09$ & $3.04 \pm 1.73 *$ \\
\hline & & Total DSTT & $12.32 \pm 2.78$ & $8.90 \pm 2.83$ \\
\hline \multirow[t]{4}{*}{ Attention test } & \multirow[t]{2}{*}{$\begin{array}{l}\text { Letter cancellation test } \\
\text { (LCT) }\end{array}$} & $\begin{array}{l}\text { Correct response } \\
\text { (LCTC) }\end{array}$ & $117 \pm 2.67$ & $111.96 \pm 6.23$ \\
\hline & & Time(second) & $100 \pm 15.03$ & $130 \pm 37.9 *$ \\
\hline & \multirow[t]{2}{*}{ Trail making test (TMT) } & TMTA (second) & $55 \pm 14$ & $76.71 \pm 25.87$ \\
\hline & & TMTB (second) & $120 \pm 20$ & $181.75 \pm 49.89^{*}$ \\
\hline \multirow{3}{*}{$\begin{array}{l}\text { Cognition test } \\
\text { (Stroop test) }\end{array}$} & Part-D (second) & & $13.12 \pm 5.55$ & $15.86 \pm 5.50$ \\
\hline & Part-W (second) & & $15.07 \pm 3.00$ & $17.08 \pm 2.32$ \\
\hline & Part-C (second) & & $18.08 \pm 2.84$ & $21.87 \pm 2.00 *$ \\
\hline
\end{tabular}

$\mathrm{SD}=$ Standard deviation; $*$ Statistically significant where $\mathrm{p}<0.05$.

\section{Results}

Test of memory: The memory status of the participants was measured by logical memory test and digit span test shown in Table 2. A significant difference was observed between premenopausal and postmenopausal women on their memory status. No significant difference was found in the immediate memory (LM-1) assessment and forward test of digit span. The result of LM-II in logical memory test, Digit span test (Backward) showed a significant difference $(\mathrm{p}<0.05)$ between premenopausal and postmenopausal women.

Attention test: The result of the Letter cancellation test (LCT) and trail making test (TMT) are used to measure the attention level of the participants was depicted in Table 2. The Trail-A making test and the number of correct responses in letter cancellation tests showed no significant differences between premenopausal and postmenopausal women. A significant difference was found in the time taken to cancel all the letters in the letter cancellation test and the Trail B making test, where the $\mathrm{p}$-value is $\mathrm{p}<0.05$. 
Cognition test: The cognitive performance of the participants evaluated by stroop test revealed a significant difference between premenopausal and postmenopausal women, shown in Table 2. No significant difference was found in the score of part $\mathrm{D}$ and part $\mathrm{W}$. The score of part $\mathrm{C}$ in stroop test showed a significant difference $(\mathrm{p}<0.05)$ between premenopausal and postmenopausal groups.

Comparison of oestradiol level: Serum oestradiol level in premenopausal and postmenopausal group shown in Figure 1. The oestradiol concentration was significantly lower $(\mathrm{p}<0.001)$ in postmenopausal group then premenopausal group.

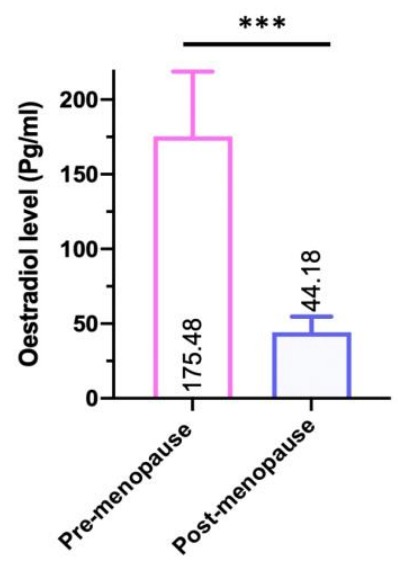

Figure 1. Mean Oestradiol level in pre and postmenopausal women $(n=15)$. Data represent the mean \pm SD.

\section{Discussion}

The present study was conducted to assess memory, attention, and cognition function between pre and postmenopausal groups and investigate the relationship between estrogen and postmenopausal memory status. The number of correct responses in LM-I, digit span test (forward test), number of correct responses in Letter Cancellation test, TMT-A, the score of Part D and Part W in Stroop test was not significant between the two groups. Other tasks, namely LM-II in logical memory test; Digit Span test (Backward); time is taken to cancel all the letters in the letter cancellation test; the TMT-B in trail making test and the score of part $\mathrm{C}$ in stroop test become significantly difference between pre and postmenopausal groups. The oestradiol concentration was significantly lower $(\mathrm{p}<0.001)$ in postmenopausal women than premenopausal women group.

Many studies reported the crucial global issue regarding the impact of estrogen on cognitive function (Lacreuse, 2006; Luine, 2008; Markou et al., 2007). Alteration of the limbic system during post menopause is allied to mood changes, anxiety, depression, insomnia, headaches, and cognitive functions (Genazzani et al., 2002). Animal studies showed that estradiol decreases anxiety and depression-like behavior in ovariectomized (OVX) rats (Walf et al., 2006). Various important neurotransmitter systems such as acetylcholine (Ach) catecholamines, serotonin and GABA are regulated by egtrogen in both human and animals (Amin et al., 2006; Dumas et al., 2006). Enhancement of the dendritic spine density on hippocampal CA1 neurons was observed after acute administration of estrogen. Furthermore, estrogen increases the concentration of Choline acetyltransferase (ChAT), involved in memory function, which is significantly decreased in Alzheimer's diseases (AD) and also inhibits AchE, and thus enhancing learning and memory (Lipatova and Toufexis, 2013).

Estrogenic actions on cognition are mediated by binding nuclear intracellular receptor estrogen receptor alpha (ER-alpha) and estrogen receptor beta (ER-beta) on basal forebrain, hippocampus, cerebral cortex, midbrain raphe, and spinal cord. Moreover, estrogen decreases the generation of oxygen free radicals by stabilizing membrane potential and prevent adenosine triphosphate (ATP) depletion (Wang et al., 2001). Estrogen regulates gene expression and modulates signaling pathways by the activation of CREB, GABA-A receptors, NMDA receptors, glutamic acid decarboxylase (GAD), ChAT and synaptic-associated proteins (Frick et al., 2002; McEwen et al., 2001; Rudick and Woolley, 2003).

In this study, we found that there was a difference in memory, attention, cognition, and estradiol level in pre and postmenopausal women. 
Memory and cognitive dysfunction in postmenopausal women were because of the sharp decline of estrogen after menopause. Apart from the estrogenic action in the hypothalamus affecting ovulation and reproductive behavior, it is evident that the brain is one of the organs that suffer significantly from the loss of estrogen after surgical or natural menopause.

\section{Conclusions}

Memory, attention, and cognitive dysfunction is a psychopathological condition most commonly occurred after menopause. The result of this study will be worthwhile to justify menopause-related memory decline and cognitive dysfunctions. In this study, we had a smaller number of samples and therefore proposing more investigation with a higher number of populations. Dietary supplements or developing a new drug that targets the estrogen receptor in the brain might be a way of treating memory decline and cognitive dysfunctions and decrease the suffering of women after menopause. The results of this study will also be able to draw interest in the scientific community for research on this specific topic further to develop a necessary clinical intervention.

\section{References}

Ali, B.H. 2004. The effect of nigella sativa oil on gentamicin nephrotoxicity in rats. The American $J$. Chinese Med. 32, 49-55.

Amin, Z., Mason, G.F., Cavus, I., Krystal, J.H., Rothman, D.L. and Epperson, C.N. 2006. The interaction of neuroactive steroids and GABA in the development of neuropsychiatric disorders in women. Pharmacol. Biochem. Behav. 84, 635-643.

Benton, A.L. 1968. Differential behavioral effectsin frontal lobe disease. Neuropsychol. 6, 53-60.

Betti, S., Orsini, M.R., Sciaky, R., Cristini, C., CesaBianchi, G. and Zandonini, G.F. 2001. Attitudes towards menopause in a group of women followed in a public service for menopause counseling. Aging. 13, 331-338.

Cersosimo, M.G. and Benarroch, E.E. 2015. Estrogen actions in the nervous system. Neurology. 85, 263273.
Clausen, A., Xu, X., Bi, X. and Baudry, M. 2012. Effects of the Superoxide Dismutase Catalase Mimetic EUK-207 in a Mouse Model of Alzheimer's Disease: Protection Against and Interruption of Progression of Amyloid and Tau Pathology and Cognitive Decline. $J$. Alzheimer's Dis. 30, 01-03.

Daniel, J.H, Aleisha, H.S., Elizabeth, S.M., Lisa, M.G. and John, C.M. 2011. Estimation of estradiol in mouse serum samples: evaluation of commercial estradiol immunoassays. Endocrinology, 152, 4443-4447.

Davey, D.A. 2013. Alzheimer's disease, dementia, mild cognitive impairment and the menopause: a 'window of opportunity'. Womens Health (L. Engl.). 9, 279290.

Dhillon, H.K., Singh, H.J., Shuib, R., Hamid, A.M. and Mahmood, N. 2006. Prevalence of menopausal symptoms in women in Kelantan, Malaysia. Maturitas. 54, 213-221.

Dumas, J., Hancur-Bucci, C., Naylor, M., Sites, C. and Newhouse, P. 2006. Estrogen treatment effects on anticholinergic-induced cognitive dysfunction in normal postmenopausal women. Neuropsychopharmacol. 31, 2065-2078.

Frick, K.M., Fernandez, S.M. and Bulinski, S.C. 2002. Estrogen replacement improves spatial reference memory and increases hippocampal synaptophysin in aged female mice. Neuroscience, 115, 547-558.

Genazzani, A.R., Monteleone, P. and Gambacciani, M. 2002. Hormonal influence on the central nervous system. Maturitas. 43, S11-S17.

Gibson, G.E. and Huang, H.M. 2005. Oxidative stress in Alzheimer's disease. Neurobiol. Aging. 26, 575-578.

Graf, P., Uttl. B. and Tuokk. H. 1995. Color and picture word stroop tests: Performance changes in old age. $J$. Clin. Exp. Neuropsychol. 17, 390-415.

Henderson, V.W. 2000. Hormone therapy and the brain: a clinical perspective on the role of estrogen. Parthenon New York.

Lacreuse, A. 2006. Effects of ovarian hormones on cognitive function in nonhuman primates. Neurosci. 138, 859-867.

Lipatova, O. and Toufexis D.J. 2013. Estrogen enhances the retention of spatial reference memory in the open field tower task but disrupts the expression of spatial memory following a novel start position. Neurobiol Learn Mem. 99, 50-58.

Liu, H. and Zhang, J. 2012. Cerebral hypoperfusion and cognitive impairment: the pathogenic role of vascular oxidative stress. Int. J. Neurosci. 122, 494-499. 
Luine, V.N. 1985. Estradiol increases choline acetyltransferase activity in specific basal forebrain nuclei and protection areas of female rats. Exp. Neurol. 89, 48490.

Luine, V.N. 2008. Sex steroids and cognitive function. $J$. Neuroendocrinol. 20, 866-872.

MacLeod, C.M. 1991. Half a century a century of research on the stroop effect: An integrative review. Psychological Bull. 109, 163-203.

Maki, P.M. and Resnick, S.M. 2000. Longitudinal effects of estrogen replacement therapy on PET cerebral blood flow and cognition. Neurobiol. Aging. 21, 373383.

Markou, A., Duka, T. and Prelevic, G.M. 2007. Estrogens and brain function. Hormones (Athens). 4, 9-17.

McEwen, B., Akama, K., Alves, S., Brake, W.G., Bulloch, K., Lee, S., Li, C., Yuen, G. and Milner T.A. 2001. Tracking the estrogen receptor in neurons: Implications for estrogen-induced synapse formation. PNAS. 13, 7093-7100.

Persad, C.C., Zubieta, J.K., Love, T., Wang, H., Tkaczyk, A. and Smith, Y.R. 2009. Enhanced neuroactivation during verbal memory processing in postmenopausal women receiving short-term hormone therapy. Fertil. Steril. 92, 197-204.

Plassman, B.L., Langa, K.M., Fisher, G.G., Heeringa, S.G. and Weir, D.R., Ofstedal, M.B., Burke, J.R., Hurd, M.D., Potter, G.G., Rodgers, W.L. and Steffens, D.C. 2007. Prevalence of dementia in the United States: the aging, demographics, and memory study. Neuroepidemiol. 29, 125-132.

Regard, M. 1981. Cognitive rigidity and flexibility: A neuropsychological study. Unpublished Ph.D. dissertation, University of Victoria.

Reitan, R.M. and Wolfson, D. 1985. The halstead-Reitan Neuropsychological Test Bettery. Neuropsychology Press, Tucson, Ariz.

Rudick, C.N. and Woolley, C.S. 2003. Selective estrogen receptor modulators regulate phasic activation of hippocampal CA1 pyramidal cells by estrogen. Endocrinology. 144, 179-187.

Small, G.W., Kepe, V., Ercoli, L.M., Siddarth, P., Bookheimer, S. ., Miller, K.J., Lavretsky, H., Burggren, A.C., Cole, G.M., Vinters, H.V. and Thompson, P.M. 2006. PET of brain amyloid and tau in mild cognitive impairment. N. Engl. J. Med. 355, 2652-2663.
Shughrue, P.J., Scrimo, P.J. and Merchenthaler, I. 2000. Estrogen binding and estrogen receptor characterization in the cholinergic neurons of rat basal forebrain. Neuroscience. 96, 41-49.

Stroop, J.R. 1935. Studies of interference in serial verbal reaction. J. Exp. Psychol. 18, 643-662.

Stuchbury, G. and Münch, G. 2005. Alzheimer's associated inflammation, potential drug targets and future therapies. J. Neural Transmission. 112, 429-453.

Tang, M.X., Jacobs, D., Stern, Y., Marder, K., Schofield, P., Gurland, B., Andrews, H. and Mayeux, R. 1996. Effect of oestrogen during menopause on risk and age at onset of Alzheimer's disease. The Lancet. 348, 429432.

Verhaeghen, P. and De Meersman. K. 1998. Aging and stroop effect: A meta-analysis. Psychol. Aging. 13, 120-126.

Walf, A.A. and Frye, C.A. 2006. A review and update of mechanisms of estrogen in the hippocampus and amygdala for anxiety and depression behavior. Neuropsychopharmacol. 31, 1097-1111.

Wang J., Green, P.S. and Simpkins, J.W. 2001. Estradiol protects against ATP depletion, mitochondrial membrane potential decline and the generation of reactive oxygen species induced by 3-nitroproprionic acid in SK-N-SH human neuroblastoma cells. $J$. Neurochem. 77, 804-11.

Wechsler, D. 1987. Manual for the Wechsler adult intelligence scale-revised. The Psychological Corporation, New York. pp. 29-31.

Wechsler, D. 1997. Administration and scoring manual for the Wechsler Adult Intelligence Scale ( ${ }^{\text {rd }}$ ed.). The Psychological Corporation, San Antonio. pp. 19-42.

Woods, N.F., Mitchell, E.S. and Adams, C. 2000. Memory functioning among midlifewomen: observations from the Seattle MidlifeWomen's Health Study. Menopause. 7, 257-65.

Xie, T., Wang, W.P., Mao, Z.F., Qu, Z.Z., Luan, S.Q., Jia, L.J. and Kan, M.C. 2012. Effects of epigallocatechin3-gallate on pentylenetetrazole-induced kindling, cognitive impairment and oxidative stress in rats. Neurosci. Lett. 516, 237-241.

Zandi, P.P., Carlson, M.C., Plassman, B.L., WelshBohmer, K.A., Mayer, L.S., Steffens, D.C. and Breitner, J.C. Cache County Memory Study Investigators, 2002. Hormone replacement therapy and incidence of Alzheimer disease in older women: the cache county study. Jama. 288, 2123-2129. 